\title{
The Interplay between Maternal Nutrition and Stress during Pregnancy: Issues and Considerations
}

\author{
Karen L. Lindsay ${ }^{a}$ Claudia Buss $^{\mathrm{a}, \mathrm{b}}$ Pathik D. Wadhwa ${ }^{a}$ Sonja Entringer ${ }^{\mathrm{a}, \mathrm{b}}$ \\ a UC Irvine Development, Health and Disease Research Program, University of California, Irvine, CA, USA; ${ }^{\text {b } C h a r i t e ́ ~-~}$ \\ Universitätsmedizin Berlin, Corporate Member of Freie Universität Berlin, Humboldt-Universität zu Berlin, and Berlin \\ Institute of Health (BIH), Institute of Medical Psychology, Berlin, Germany
}

\section{Keywords}

Pregnancy $\cdot$ Nutrition $\cdot$ Dietary intakes $\cdot$ Psychosocial states . Perceived stress · Anxiety · Depression · Fetal programming

\begin{abstract}
Background: Several studies about humans and animals have separately examined the effects of prenatal nutrition and stress on fetal development, pregnancy, and birth outcomes, and subsequent child health and disease risk. Although substantial evidence from non-pregnant literature supports the presence of bidirectional interactions between nutrition and stress at various psychological, behavioral, and physiological levels, such interaction effects have not yet been systematically examined in the context of pregnancy. Summary: This paper discusses the multifaceted and multilevel relationship between nutrition and stress. It then reviews the currently available observational and experimental evidence in animals and humans regarding the interplay between maternal psychosocial stress, dietary intake, and nutritional state during pregnancy, and implications for maternal and child health-related outcomes. Key Messages: During pregnancy, maternal psychosocial stress, dietary behavior, and nutritional state likely regulate and counter-regulate one another. Emerging evidence suggests that omega-3 fatty acids may attenuate maternal psychosocial stress, and that high maternal pre-pregnancy body mass index ex-
\end{abstract}

\section{KARGER}

(c) 2017 S. Karger AG, Basel

E-Mail karger@karger.com

www.karger.com/anm acerbates unhealthy dietary behaviors under high-stress conditions. Longitudinal studies are warranted in order to understand the interplay between prenatal psychosocial stress, diet, and stress- and nutrition-related biomarkers to obtain further insight and inform the development and design of future, more effective intervention trials for improved maternal and child health outcomes.

(c) 2017 S. Karger AG, Basel

\section{Introduction}

Substantial evidence suggests that conditions during intrauterine life play a major role in shaping not only all aspects of fetal development and birth outcomes but also subsequent newborn, child, and adult health outcomes and susceptibility for many of the complex, common disorders that confer the major burden of disease in society (i.e., the concept of fetal, or developmental, origins of health and disease risk) [1-4]. In this context, 2 of the most frequently studied factors in pregnancy are maternal stress and maternal nutrition. The vast majority of these studies have investigated only one or the other but not both of these factors concurrently, and the few studies that have included both have treated one of the factors as the primary variable of interest and the other as a potential confounder/covariate. It is now increasingly apparent

Sonja Entringer, $\mathrm{PhD}$

Institut für Medizinische Psychologie

Charité Centrum für Human- und Gesundheitswissenschaften

Charité Universitätsmedizin Berlin, Luisenstrasse 57, DE-10117 Berlin (Germany)

E-Mail sonja.entringer@charite.de 
that the causation of complex, common disorders could not and in fact does not reside in any single risk factor or in the mere additive effects of numerous factors, but likely lies at the interface between multiple risk factors (interaction, or multiplicative effects) $[5,6]$. Growing evidence supports the existence of interactions between stress- and nutrition-related processes at multiple levels across and over time; however, this issue has not yet been adequately addressed in the pregnancy and fetal programming literatures.

The importance of concurrently considering prenatal stress and nutrition interactions is based, in part, on concepts from evolutionary biology and developmental plasticity. Development describes the journey from genotype to phenotype, and developmental plasticity describes the process of phenotypic specification (of structure and function) conditioned upon genotype and environment. Key environmental conditions that have shaped evolutionary selection and developmental plasticity include variation in energy substrate availability (i.e., nutrition) and other challenges that have the potential to impact the structural or functional integrity and survival of the organism (i.e., stress) $[7,8]$. Thus, it is likely and plausible that prenatal stress and nutrition interactions represent an important aspect of the intrauterine environment that would be expected to influence many, if not all, developmental outcomes [9-11]. This formulation is consistent with empirical data in humans and animals. Variation in maternal nutrition (e.g., maternal over- and undernutrition, variation in dietary quality, micronutrient intake) is associated with offspring health- and disease risk-related outcomes [12-17]. Variation in maternal stress and stress-related biological processes during pregnancy is also associated with a multitude of developmental and health outcomes in the offspring $[6,7,18-22]$. Furthermore, evidence from studies in non-pregnant individuals demonstrates the substantial intertwining of the relationship between stress, dietary behavior, and nutritional biochemistry [23-25].

Addressing the role of prenatal conditions that may impact phenotypic specification is critical to optimize or improve the health of future generations [13]. Pregnancy is often described as a "window of opportunity" to promote healthful maternal states and behaviors for the benefit of the offspring $[26,27]$. However, many studies focusing on prenatal dietary and/or exercise interventions fail to consider maternal stress or other psychological states, which may impact intervention effectiveness due to poor motivation, self-efficacy, or altered metabolic pathways $[28,29]$. This paper first discusses the multifac- eted relationship between nutrition and stress in humans. It then reviews the evidence present in observational studies in animals and humans that have investigated the interplay between maternal stress and dietary intake during pregnancy, and the implications thereof for adverse outcomes relating to maternal and child metabolic health. Lastly, it reviews findings from intervention trials that have utilized nutritional components to alter psychosocial or biological stress parameters in pregnancy.

\section{The Interplay between Nutrition and Stress}

Evidence from studies of non-pregnant humans strongly supports the presence of a recursive, bidirectional relationship between nutrition and stress [23-25]. It is important to appreciate that "nutrition" and "stress" are broad terms that may be operationalized in various ways. Nutrition may refer to an individual's biophysical status (ranging from underweight to obesity), nutrient status within the body (e.g., circulating fatty acids), dietary intake of foods and nutrients, and metabolic response to ingested foods (e.g., digestive processes). Similarly, stress has 3 key components: the occurrence of potentially stressful situations or events, the individual's perception of the nature and magnitude of distress following such events or regarding chronic life circumstances (perceived stress), and the consequent psychological (e.g., depression, anxiety) or biological (e.g., stress hormone concentrations) responses. Thus, studies examining nutritionstress associations may describe interactions across various combinations of these different components. For example, Groesz et al. [30] found that among women, perceptions of higher chronic stress exposure affected dietary intake, leading to greater hunger, binge-eating, and intake of palatable non-nutritious foods. Poor diet quality, with preponderance for consumption of unhealthy foods high in fat and sugar, is frequently reported across various cohorts in relation to perceived and/or experimental stress induction [31-35]. Experimental challenges of exposure to psychological stress have been shown to alter the digestive and metabolic responses to ingested food, including delayed gastric emptying, slower motility and transit throughout the gastrointestinal tract [36], reduced rate of triglyceride clearance from the blood [37, 38], and disturbed postprandial regulation of insulin [39]. Moreover, stress-induced disturbances of normal digestion and metabolism may ultimately influence the metabolic fate of nutrients in target organs and tissues. For example, moderately elevated levels of circulating cortisol 
(as seen in chronic stress conditions) amplify insulin action on the liver by increasing liver gluconeogenesis and lipogenesis [40]. In adipose tissue, insulin and cortisol stimulate adipocyte differentiation and lipid deposition via elevated lipoprotein lipase activity [41]. Similarly, the nutritional state can influence the stress response at multiple levels. Nutritional status may alter the perception of the degree of stressfulness of an event or situation, thus modulating the biological stress hormone cascade. As mentioned above, psychosocial stress tends to increase the desire to consume palatable foods high in fat and sugar, the intake of which subsequently dampens the stress response, reduces feelings of anxiety and distress, and thus leads to "emotional eating" [42, 43].

Furthermore, it is also recognized that specific nutrients play a critical role in modulating mood, stress, and development of psychological disorders. In this regard, polyunsaturated fatty acids (PUFAs) have received particular attention due to their multiple roles in brain function, including the modification of membrane fluidity, membrane enzyme activity, the number and affinity of receptors, the function of neuronal membrane ionic channels, and the production of neurotransmitters and ionic peptides [44]. The long-chain omega-3 docosahexanoic acid (DHA), which is primarily found in oily fish, appears to be the most critical of the PUFAs due to its high concentration in phospholipids of neural cells [45]. Its incorporation in the brain occurs almost exclusively in perinatal life [46], and consequently, DHA deficiency during this critical stage of early neurodevelopment has been associated with heightened vulnerability toward depression, aggression, and stress in later life [47]. Dietary intake of omega-3 fatty acids throughout life remains important to replenish the daily loss of brain DHA [48, 49], thereby maintaining normal neurological function. Dietary omega-3 may also increase adaptation to chronic stress, as demonstrated in a study in which rats fed an omega-3-enriched diet experienced less stress-induced weight loss and a lower plasma corticosterone peak compared to rats fed either a standard control or an omega3 -deficient diet [50]. This study also found that the omega-3-deficient rats exhibited a significantly greater startle response to a stressor, highlighting the role of omega-3 fatty acids in modulating behavioral responses in addition to stress-related physiological and biochemical processes. Indeed, reduced dietary intake of oily fish, commonplace across many Western populations today, is associated with increased prevalence of anxiety and depressive disorders $[51,52]$. Deficiencies in various micronutrients, including B-complex vitamins, vitamin D,

Nutrition-Stress Interactions in Pregnancy zinc, chromium, and iodine, have also been found to be associated with stress-related disorders like depression, anxiety, and other neuropsychiatric disorders [53], and prenatal supplementation of such nutrients may prevent many brain and central nervous system impairments in the offspring [54].

\section{Nutrition-Stress Interactions during Pregnancy}

Based on the evidence reviewed above, it is evident that the relationships between stress, diet, and nutrition are complex and multifaceted. In the context of pregnancy and fetal development, although nutrition and stress have been separately studied for their effects on maternal and child health outcomes, relatively few studies have considered their combined or interactive effects. Due to the limited number of published studies and heterogeneity in study design across both animal and human studies on the topic of nutrition-stress interactions during pregnancy, a systematic review was not feasible. To provide an overview of the available literature on this topic, we instead performed a comprehensive literature review across several online databases (PubMed, Ovid, Science Direct) to identify English language publications describing experimental, observational, and intervention studies addressing the interplay between nutrition and stress in the perinatal period in animals or humans. No limit on the date of publication was applied to the literature search and all studies that presented data on any aspect of psychosocial stress in conjunction with any diet-related measure in pregnancy were considered.

\section{Animal Studies}

Although a large number of experimental studies in animals have examined the effects of stress induction during pregnancy on offspring metabolic health and obesity risk [55-60], we were able to identify only a couple of studies that addressed the issue of stress-nutrition interactions. Amugongo and Hlusko [61] reported that rats exposed to stress in early but not late gestation displayed a $12.5 \%$ reduction in daily food intake compared to controls, resulting in reduced weight gain in the early gestation stressed animals. Tamashiro et al. [57] examined the separate and combined effects in rats; experimentally-altered prenatal stress and diet on both maternal and offspring metabolic health outcomes were examined. Pregnant rats were fed either standard chow or high-fat diet, and half the number of rats of each group were subjected to a variable stress exposure paradigm over several days 
in late gestation. Regarding maternal outcomes, only the high-fat-stress group experienced significantly higher body weight gain during gestation compared to the control group (standard chow, no prenatal stress), supporting the importance of studying stress by nutrition interactions. Among the neonatal offspring, those born to mothers fed a high-fat diet with or without stress exposure had higher fasting glucose, insulin, and leptin, while in adulthood, female offspring exposed to prenatal high-fat-stress had greater percent body fat compared to the standardchow-control offspring. Furthermore, pups weaned onto a high-fat diet were observed to have impaired glucose tolerance if exposed to stress and/or high-fat diet during gestation [57]. While this experimental animal model of prenatal stress and dietary manipulation demonstrates various adverse metabolic outcomes in offspring, the effects appear to vary in terms of offspring sex and postnatal diet, and it is not clear whether the combination of prenatal stress and high-fat diet produces consistently worse outcomes in the offspring compared to one of the exposures in isolation. Furthermore, it is difficult to extrapolate such results from rodents to humans, particularly given that the composition of a standard "high-fat" experimental rodent diet (e.g., 60\% energy from fat derived entirely from lard and soybean oil) is highly unrepresentative of human dietary intake, regardless of phenotype (e.g., obesity) or other exposures (e.g., psychosocial stress) $[62,63]$. Thus, there is a compelling need for human pregnancy studies examining the interactions among prenatal stress, dietary behaviors, and nutrient state.

\section{Human Studies}

As summarized in Table 1, we identified 8 observational studies examining stress-nutrition associations in human pregnancy. Each of these studies focused only on maternal outcomes and did not extend to the postnatal period, thus precluding the possibility of studying fetal programming effects in the offspring. The majority of these studies were cross-sectional in design, evaluating dietary intake of specific nutrients, food groups, broader dietary patterns, or eating behaviors at a single time point in pregnancy, and then examining their relationship with various psychosocial outcomes.

In the earliest published study, Hurley et al. [64] administered several validated psychosocial questionnaires and a food frequency questionnaire to healthy pregnant women in mid-gestation. Higher overall energy intake, which is likely due to the result of greater consumption of the "fats, oils, sweets, and snack" food group, correlated with higher ratings of perceived stress, which persisted after adjusting for maternal body mass index (BMI), age, parity, and education. These findings corroborate those in non-pregnant humans associating increased consumption of energy-dense, palatable, unhealthy foods with psychosocial stress [31, 32]. More recently, Chang et al. [65] used path analysis techniques in a cohort of low-income overweight and obese pregnant women and identified maternal depression as a mediator of the positive relationship between dietary fat intake and perceived stress in the first but not second and third trimesters of gestation. Increased maternal BMI in pregnancy has previously been associated with unhealthy dietary patterns and suboptimal nutrient intake [66], whereas a low socioeconomic status is associated with increased psychosocial stress, depression, and anxiety among both non-pregnant and pregnant women $[67,68]$. However, one limitation of the Chang et al. [65] study is that they only assessed diet using a Rapid Food Screener tool, which limited dietary intake questions only to the intake of fruit and vegetables and high-fat foods (with no specification for type or quality of dietary fat); thus, they were unable to identify more nuanced associations between psychosocial state and specific food and nutrient intakes. In particular, they were unable to investigate the differential effects of stress on intake of fatty acid subtypes, which may be particularly relevant, given the important role of omega-3 PUFAs in early neurological development, prevention of depression, and optimal adaptation to stress $[47,51,52]$. Indeed, 2 reports from a very large pregnancy cohort found that women with low or no dietary intake of omega-3 PUFA from fish experienced significantly higher levels of anxiety [69] and depression symptoms [70] during pregnancy.

In another cross-sectional study of pregnant women in the third trimester, Goncalves et al. [71] identified various maternal traits (e.g., emotional problems), states (e.g., higher BMI), and attitudes (e.g., negative affect towards the baby) that correlated with dysfunctional eating behaviors. In this study, it was the combination of increased maternal BMI, greater gestational weight gain, and negative perception of body image that explained greatest variation $(32 \%)$ in the eating disorder global score. However, given the cross-sectional nature of the study, it was not possible to ascertain whether one set of exposures preceded the other. Based on empirical, observational, and experimental evidence from the non-pregnancy literature [31-35], we propose that a vicious cycle of psychosocial stress and eating behavior may manifest among susceptible women during pregnancy. For example, in the study by Goncalves et al. [71], negative emotions and 


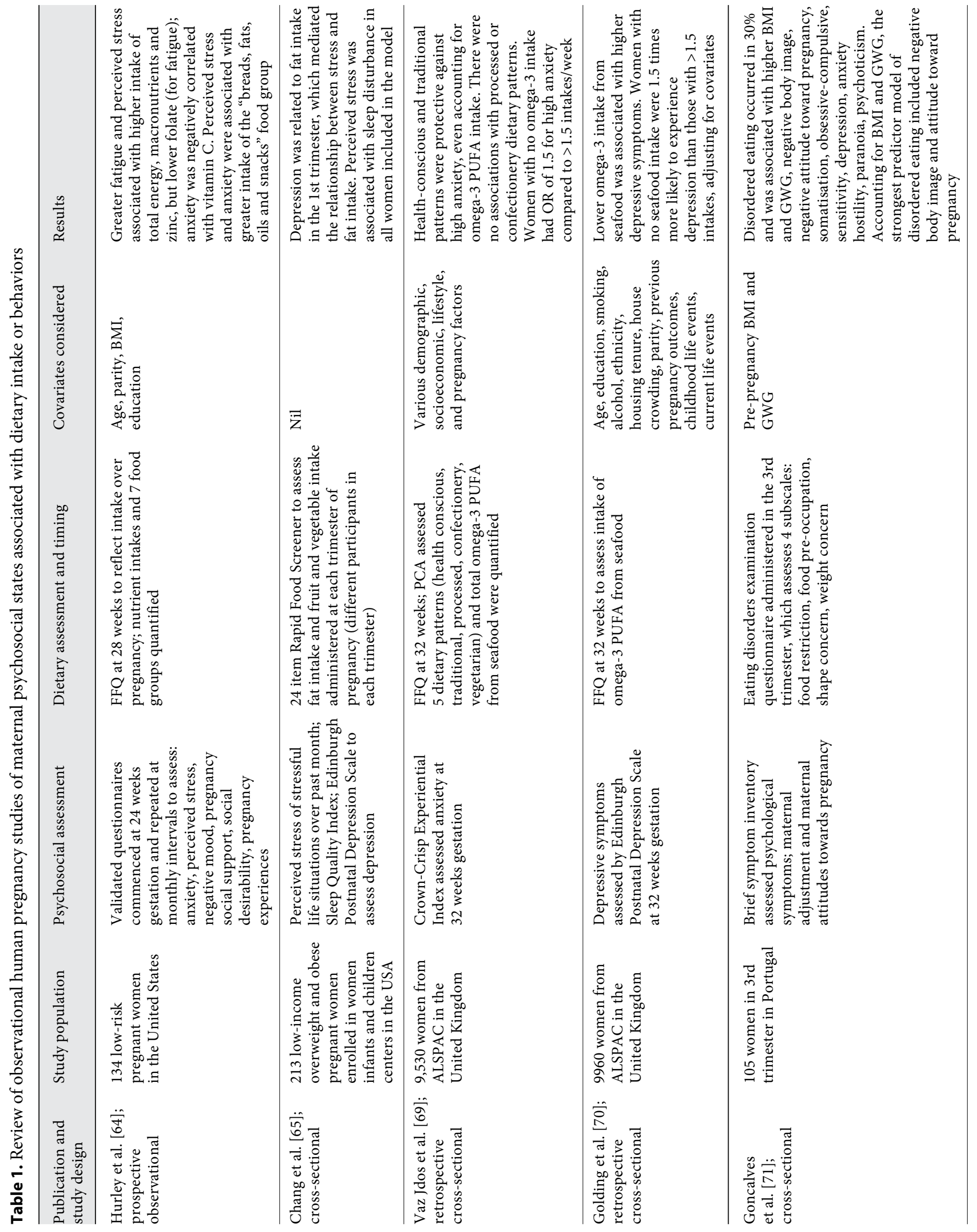




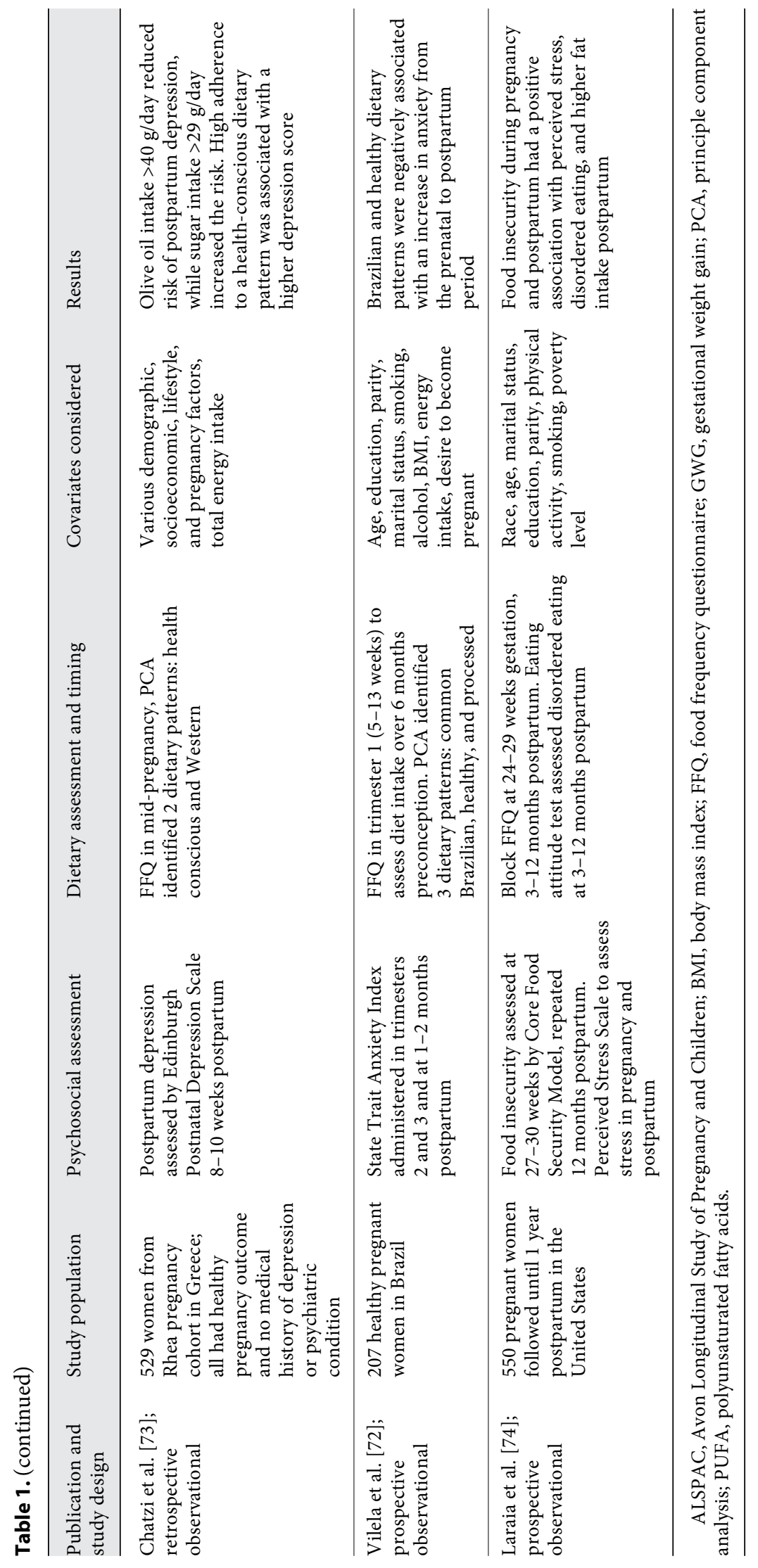


attitudes toward pregnancy may alter biological stress responses that influence unhealthy food selection and poor overall dietary quality as an "emotional eating" coping mechanism, subsequently leading to increased gestational weight gain, re-enforcing the negative perception of body image and disordered eating behaviors, and, in turn, exacerbating the negative emotions and psychosocial states.

Prospective studies over the prenatal and postpartum periods have attempted to examine maternal nutritionstress associations on the basis of broader dietary patterns. In a cohort of Brazilian pregnant women, Vilela et al. [72] reported that adherence to pre-pregnancy "traditional Brazilian" and "healthy" dietary patterns was inversely associated with state trait anxiety scores across pregnancy and into the postpartum period. Similarly, in a Mediterranean Greek pregnant population, higher maternal adherence to the "health-conscious" dietary pattern in midpregnancy was associated with lower risk of postpartum depression symptoms, after adjusting for a wide range of potential confounding factors [73]. The authors postulated that among foods contributing to the health-conscious dietary pattern in this study (vegetables, fruit, pulses, nuts, dairy products, oily fish, and olive oil), the high intake of omega-3 PUFAs from oily fish and potent antioxidant compounds (carotenoids, alpha-tocopherol and polyphenols) present in the monounsaturated fatty acid-rich olive oil, were significant contributors to the reduction in depressive symptoms, thus corroborating with the findings of Golding et al. [70] and Vaz Jdos et al. [69].

Lastly, Laraia et al. [74] conducted a prospective study of the impact of food insecurity during pregnancy on psychosocial and diet-related outcomes in the postpartum period. Food insecurity is a multidimensional construct that captures anxiety associated with the uncertainty of being able to obtain, purchase, and consume an adequate diet due to financial and socioeconomic constraints, often accompanied by a dependence on low-cost, caloriedense foods, and disordered eating patterns such as binge eating [75]. This study found that higher food insecurity was a consistent and independent predictor of maternal postpartum perceived stress, negative eating attitudes, and dietary intake of total fat as a percentage of energy intake [74]. Furthermore, higher maternal prepregnancy BMI was positively associated with food insecurity status. Although the composition of dietary fat intake was not distinguished, it is unlikely that healthy fats such as those from oily fish and olive oil contributed to the higher fat intake among women experiencing food insecurity. This study highlights the importance of considering maternal socioeconomic status-related factors as contributors to, and/or moderators of, prenatal nutrition-stress interactions.

None of the human pregnancy studies in this review examined biochemical parameters or neonatal/child outcomes arising from observed nutrition-stress interactions. Thus, to our knowledge, there are no human pregnancy studies to date, which concurrently investigate putative psychosocial stress and nutrition interactions underlying fetal programming of offspring health and disease-related outcomes, despite strong empirical evidence for such mechanisms $[6,9,10]$.

\section{Prenatal Interventions}

There remains a paucity of interventional studies attempting to systematically test whether maternal nutrition- or stress-related states in pregnancy vary as a function of one another.

\section{Effect of Prenatal Nutrition Interventions on}

\section{Stress-Related Outcomes}

To our knowledge, only a small number of randomized controlled trials in humans have investigated the impact of specific prenatal nutritional supplementation (e.g., different fatty acid combinations) on maternal biological and psychosocial stress outcomes. We were unable to find any published trials investigating the impact of dietary-based interventions on maternal stress-related outcomes.

In a small placebo-controlled RCT in a Chinese cohort $(n=36)$, the impact of a daily omega-3 supplement taken over 8 weeks was examined on depressive symptoms among women diagnosed with severe depressive disorder during pregnancy and not treated with psychotropic medications [76]. Women randomized to the omega- 3 supplements (2.2 g EPA + 1.2 g DHA) had significantly decreased depression scores on both clinically measured and self-reported scales. Meanwhile, in a low-income African-American cohort in the United States $(n=64)$, a daily $450 \mathrm{mg}$ DHA + 15 IU Vitamin E supplement reduced women's level of perceived stress and modulated their physiological stress response (salivary cortisol concentrations) to a laboratory-based psychosocial stress paradigm [77]. Neither trial assessed dietary intakes among the cohorts, although the latter study excluded women who reported consuming greater than 2 servings of fish per week, which could have further elevated women's DHA intakes [77]. Together, these trials provide the first evidence that 
a targeted nutritional supplement intervention during pregnancy may modulate maternal stress perceptions as well as physiological stress responses.

More recently, 3-armed prenatal RCTs of lipid-based nutrition supplements (containing omega- 3 and 6 fatty acids, protein and 22 micronutrients) have been conducted in several low- and middle-income countries. In 2 of these studies among Malawian and Ghanian cohorts, maternal salivary cortisol concentrations were measured in addition to neonatal outcomes, in order to compare the effects of the lipid-based supplement to a standard multiple-micronutrient (containing 18 micronutrients) or iron-folic acid only supplements $[78,79]$. These studies did not identify any significant differences in salivary cortisol concentrations across gestation between intervention groups, indicating that the lipid-based nutrition supplement did not affect the inverse relationship between maternal cortisol and length of gestation and birthweight in this obstetric cohort at high risk for adverse neonatal outcomes, including pre-term birth and low birthweight.

\section{Effect of Prenatal Psychosocial Interventions during}

Pregnancy on Nutrition or Metabolic Outcomes

To our knowledge, there are currently no published studies investigating the impact of a psychosocial intervention during pregnancy on maternal dietary behaviors or other nutrition-related outcome. However, we identified one open trial registered on ClinicalTrials.gov (NCT01307683), which will utilize a mindfulness-based eating and stress-reduction program with the aim of reducing gestational weight gain among low-income, overweight pregnant women. The researchers from this trial have published the findings of a pilot qualitative study exploring the target population's perception of such an intervention [80]. Overall, women strongly identified with struggles relating to the complex interplay of emotions, stress, and eating behaviors in their lives, expressed concern regarding the impact of their stress and dietary behaviors on weight gain and health of their developing babies, and were enthusiastic and optimistic about a stress-reduction intervention with group support to provide meaningful skills and target the emotional components of eating during pregnancy.

\section{Conclusions}

Maternal dietary intake, nutritional status, and psychosocial states and conditions during pregnancy are likely to play critical roles in influencing maternal meta- bolic health, fetal development, and offspring health outcomes; yet the complex relationships and interactive effects between these factors have not yet been studied in humans or animals. The small number of studies identified in this review that examined such interactions support the concept that prenatal psychosocial stress is associated with unhealthy dietary intakes and eating behaviors, such as "comfort eating" of highly palatable foods. It appears likely that women who become pregnant with an increased BMI may be more susceptible to unhealthy dietary behaviors potentially driven by poor psychosocial states, which may exacerbate the already elevated risk for adverse pregnancy and infant outcomes related to maternal obesity. With respect to specific dietary components, emerging evidence from human pregnancy studies suggests that omega-3 PUFA may play a critical role in not only fetal neurodevelopment, but also for supporting positive maternal mood and decreasing the risk of unfavorable psychological states such as perceived stress, anxiety, and depression in the pre- and postnatal periods. Although the mechanisms underlying the effects of omega-3 PUFA during pregnancy on moderating these outcomes remain to be elucidated, it is likely associated with their multiple important roles in supporting normal brain function [44], which are perhaps compromised during pregnancy when PUFA deficiency or insufficiency occurs secondary to increased fetal demands $[81,82]$.

Longitudinal observational studies with comprehensive evaluation of maternal psychosocial stress and diet across pregnancy and stress- and nutrition-related biomarkers will likely provide better insight to the nature and magnitude of their inter-relationships. Such studies could then better inform the development of specific hypotheses and design of future, more effective intervention trials of maternal psychological state in combination with dietary and lifestyle factors for improved maternal and child health outcomes.

\section{Disclosure Statement}

The authors declare no conflicts of interest.

\section{Acknowledgments}

Support for the preparation of this manuscript was provided, in part, by US PHS (NIH) grants R01 HD-060628, R01 HD-065825, R01 MD-010738, and by the Commission of the European Communities, the 7th Framework Programme, contract FP7289346-EARLY NUTRITION. 


\section{References}

1 Barker DJ: Obesity and early life. Obes Rev 2007;8(suppl 1):45-49.

2 Armitage JA, Poston L, Taylor PD: Developmental origins of obesity and the metabolic syndrome: the role of maternal obesity. Front Horm Res 2008;36:73-84.

3 Wadhwa PD, Buss C, Entringer S, Swanson JM: Developmental origins of health and disease: brief history of the approach and current focus on epigenetic mechanisms. Semin Reprod Med 2009;27:358-368.

4 Swanson JM, Entringer S, Buss C, Wadhwa PD: Developmental origins of health and disease: environmental exposures. Semin Reprod Med 2009;27:391-402.

5 Laland KN, Sterelny K, Odling-Smee J, Hoppitt W, Uller T: Cause and effect in biology revisited: is Mayr's proximate-ultimate dichotomy still useful? Science 2011;334:15121516.

6 Entringer S: Impact of stress and stress physiology during pregnancy on child metabolic function and obesity risk. Curr Opin Clin Nutr Metab Care 2013;16:320-327.

7 Entringer S, Buss C, Swanson JM, Cooper DM, Wing DA, Waffarn F, et al: Fetal programming of body composition, obesity, and metabolic function: the role of intrauterine stress and stress biology. J Nutr Metab 2012; 2012:632548.

8 Wadhwa PD: Psychoneuroendocrine processes in human pregnancy influence fetal development and health. Psychoneuroendocrinology 2005;30:724-743.

9 Entringer S, Buss C, Wadhwa PD: Prenatal stress and developmental programming of human health and disease risk: concepts and integration of empirical findings. Curr Opin Endocrinol Diabetes Obes 2010;17:507-516.

10 Hobel C, Culhane J: Role of psychosocial and nutritional stress on poor pregnancy outcome. J Nutr 2003;133(5 suppl 2):1709S$1717 \mathrm{~S}$

11 Spencer SJ: Perinatal nutrition programs neuroimmune function long-term: mechanisms and implications. Front Neurosci 2013;7:144.

12 Horan MK, McGowan CA, Gibney ER, Donnelly JM, McAuliffe FM: Maternal low glycaemic index diet, fat intake and postprandial glucose influences neonatal adiposity - secondary analysis from the ROLO study. Nutr J 2014;13:78.

13 Dabelea D, Crume T: Maternal environment and the transgenerational cycle of obesity and diabetes. Diabetes 2011;60:1849-1855.

14 Reynolds CM, Gray C, Li M, Segovia SA Vickers $\mathrm{MH}$ : Early life nutrition and energy balance disorders in offspring in later life. $\mathrm{Nu}$ trients 2015;7:8090-8111.

15 Pet MA, Brouwer-Brolsma EM: The impact of maternal vitamin $\mathrm{D}$ status on offspring brain development and function: a systematic review. Adv Nutr 2016;7:665-678.

16 Emmett PM, Jones LR, Golding J: Pregnancy diet and associated outcomes in the Avon
Longitudinal Study of Parents and Children. Nutr Rev 2015;73(suppl 3):154-174.

17 Devakumar D, Fall CH, Sachdev HS, Margetts BM, Osmond C, Wells JC, et al: Maternal antenatal multiple micronutrient supplementation for long-term health benefits in children: a systematic review and meta-analysis. BMC Med 2016;14:90.

18 Entringer S, Buss C, Wadhwa PD: Prenatal stress, telomere biology, and fetal programming of health and disease risk. Sci Signal 2012;5:pt12.

19 Buss C, Entringer S, Wadhwa PD: Fetal programming of brain development: intrauterine stress and susceptibility to psychopathology. Sci Signal 2012;5:pt7.

20 Marques AH, O'Connor TG, Roth C, Susser E, Bjorke-Monsen AL: The influence of maternal prenatal and early childhood nutrition and maternal prenatal stress on offspring immune system development and neurodevelopmental disorders. Front Neurosci 2013;7: 120.

21 Kinsella MT, Monk C: Impact of maternal stress, depression and anxiety on fetal neurobehavioral development. Clin Obstet Gynecol 2009;52:425-440.

22 Dunkel Schetter C: Psychological science on pregnancy: stress processes, biopsychosocial models, and emerging research issues. Annu Rev Psychol 2011;62:531-558.

23 Adam TC, Epel ES: Stress, eating and the reward system. Physiol Behav 2007;91:449-458.

24 Torres SJ, Nowson CA: Relationship between stress, eating behavior, and obesity. Nutrition 2007;23:887-894.

25 Kiecolt-Glaser JK: Stress, food, and inflammation: psychoneuroimmunology and nutrition at the cutting edge. Psychosom Med 2010;72:365-369.

26 Phelan S: Pregnancy: a "teachable moment" for weight control and obesity prevention. Am J Obstet Gynecol 2010;202:135.e1-e8.

27 Kapur A: Pregnancy: a window of opportunity for improving current and future health. Int J Gynaecol Obstet 2011;115(suppl 1):S50S51.

28 Sui Z, Turnbull DA, Dodd JM: Overweight and obese women's perceptions about making healthy change during pregnancy: a mixed method study. Matern Child Health J 2013; 17:1879-1887.

29 Sui Z, Turnbull D, Dodd J: Enablers of and barriers to making healthy change during pregnancy in overweight and obese women. Australas Med J 2013;6:565-577.

30 Groesz LM, McCoy S, Carl J, Saslow L, Stewart J, Adler N, et al: What is eating you? Stress and the drive to eat. Appetite 2012;58:717721

31 Dallman MF, Pecoraro N, Akana SF, La Fleur SE, Gomez F, Houshyar H, et al: Chronic stress and obesity: a new view of "comfort food”. Proc Natl Acad Sci U S A 2003;100: 11696-11701.
32 Epel E, Lapidus R, McEwen B, Brownell K: Stress may add bite to appetite in women: a laboratory study of stress-induced cortisol and eating behavior. Psychoneuroendocrinology 2001;26:37-49.

33 Oliver G, Wardle J, Gibson EL: Stress and food choice: a laboratory study. Psychosom Med 2000;62:853-865.

34 Zellner DA, Loaiza S, Gonzalez Z, Pita J, Morales J, Pecora D, et al: Food selection changes under stress. Physiol Behav 2006;87:789-793.

35 Barrington WE, Beresford SA, McGregor BA White E: Perceived stress and eating behaviors by sex, obesity status, and stress vulnerability: findings from the vitamins and lifestyle (VITAL) study. J Acad Nutr Diet 2014; 114:1791-1799.

36 Yin J, Levanon D, Chen JD: Inhibitory effects of stress on postprandial gastric myoelectrical activity and vagal tone in healthy subjects. Neurogastroenterol Motil 2004;16: 737-744.

37 Stoney CM, West SG, Hughes JW, Lentino LM, Finney ML, Falko J, et al: Acute psychological stress reduces plasma triglyceride clearance. Psychophysiology 2002;39:80-85.

38 Le Fur C, Romon M, Lebel P, Devos P, Lancry A, Guedon-Moreau L, et al: Influence of mental stress and circadian cycle on postprandial lipemia. Am J Clin Nutr 1999;70:213-220.

39 Teff KL: Visceral nerves: vagal and sympathetic innervation. JPEN J Parenter Enteral Nutr 2008;32:569-571.

40 Siervo M, Wells JC, Cizza G: The contribution of psychosocial stress to the obesity epidemic: an evolutionary approach. Horm Metab Res 2009;41:261-270.

41 Fried SK, Russell CD, Grauso NL, Brolin RE: Lipoprotein lipase regulation by insulin and glucocorticoid in subcutaneous and omental adipose tissues of obese women and men. J Clin Invest 1993;92:2191-2198.

42 Mikolajczyk RT, El Ansari W, Maxwell AE: Food consumption frequency and perceived stress and depressive symptoms among students in three European countries. Nutr J 2009;8:31.

43 Dallman MF: Stress-induced obesity and the emotional nervous system. Trends Endocrinol Metab 2010;21:159-165.

44 Yehuda S: Omega-6/omega-3 ratio and brainrelated functions. World Rev Nutr Diet 2003; 92:37-56.

45 Alessandri JM, Guesnet P, Vancassel S, Astorg P, Denis I, Langelier B, et al: Polyunsaturated fatty acids in the central nervous system: evolution of concepts and nutritional implications throughout life. Reprod Nutr Dev 2004;44:509-538.

46 Cunnane SC, Francescutti V, Brenna JT, Crawford MA: Breast-fed infants achieve a higher rate of brain and whole body docosahexaenoate accumulation than formula-fed infants not consuming dietary docosahexaenoate. Lipids 2000;35:105-111. 
47 Hibbeln JR, Ferguson TA, Blasbalg TL: Omega-3 fatty acid deficiencies in neurodevelopment, aggression and autonomic dysregulation: opportunities for intervention. Int Rev Psychiatry 2006;18:107-118.

48 Liperoti R, Landi F, Fusco O, Bernabei R, Onder G: Omega-3 polyunsaturated fatty acids and depression: a review of the evidence. Curr Pharm Des 2009;15:4165-4172.

49 Rapoport SI, Rao JS, Igarashi M: Brain metabolism of nutritionally essential polyunsaturated fatty acids depends on both the diet and the liver. Prostaglandins Leukot Essent Fatty Acids 2007;77:251-261.

50 Hennebelle M, Balasse L, Latour A, Champeil-Potokar G, Denis S, Lavialle M, et al: Influence of omega-3 fatty acid status on the way rats adapt to chronic restraint stress. PLoS One 2012;7:e42142.

51 Jacka FN, Pasco JA, Williams LJ, Meyer BJ, Digger R, Berk M: Dietary intake of fish and PUFA, and clinical depressive and anxiety disorders in women. Br J Nutr 2013;109: 2059-2066.

52 Grosso G, Galvano F, Marventano S, Malaguarnera M, Bucolo C, Drago F, et al: Omega-3 fatty acids and depression: scientific evidence and biological mechanisms. Oxid Med Cell Longev 2014;2014:313570.

53 Rao TS, Asha MR, Ramesh BN, Rao KS: Understanding nutrition, depression and mental illnesses. Indian J Psychiatry 2008;50:77-82.

54 Morse NL: Benefits of docosahexaenoic acid, folic acid, vitamin D and iodine on foetal and infant brain development and function following maternal supplementation during pregnancy and lactation. Nutrients 2012;4: 799-840.

55 Balasubramanian P, Varde PA, Abdallah SL, Najjar SM, MohanKumar PS, MohanKumar SM: Differential effects of prenatal stress on metabolic programming in diet-induced obese and dietary-resistant rats. Am J Physiol Endocrinol Metab 2015;309:E582-E588.

56 Brunton PJ, Sullivan KM, Kerrigan D, Russell JA, Seckl JR, Drake AJ: Sex-specific effects of prenatal stress on glucose homoeostasis and peripheral metabolism in rats. J Endocrinol 2013;217:161-173.

57 Tamashiro KL, Terrillion CE, Hyun J, Koenig JI, Moran TH: Prenatal stress or high-fat diet increases susceptibility to diet-induced obesity in rat offspring. Diabetes 2009;58:1116-1125.

58 D'Mello AP, Liu Y: Effects of maternal immobilization stress on birth weight and glucose homeostasis in the offspring. Psychoneuroendocrinology 2006;31:395-406.

59 Mueller BR, Bale TL: Impact of prenatal stress on long term body weight is dependent on timing and maternal sensitivity. Physiol Behav 2006;88:605-614.
60 Lesage J, Del-Favero F, Leonhardt M, Louvart H, Maccari S, Vieau D, et al: Prenatal stress induces intrauterine growth restriction and programmes glucose intolerance and feeding behaviour disturbances in the aged rat. J Endocrinol 2004;181:291-296.

61 Amugongo SK, Hlusko LJ: Impact of maternal prenatal stress on growth of the offspring. Aging Dis 2013;5:1-16.

62 Lai M, Chandrasekera PC, Barnard ND: You are what you eat, or are you? The challenges of translating high-fat-fed rodents to human obesity and diabetes. Nutr Diabetes 2014; 4:e135.

63 Warden $\mathrm{CH}$, Fisler JS: Comparisons of diets used in animal models of high-fat feeding. Cell Metab 2008;7:277.

64 Hurley KM, Caulfield LE, Sacco LM, Costigan KA, Dipietro JA: Psychosocial influences in dietary patterns during pregnancy. J Am Diet Assoc 2005; 105:963-966.

65 Chang MW, Brown R, Nitzke S, Smith B, Eghtedary K: Stress, sleep, depression and dietary intakes among low-income overweight and obese pregnant women. Matern Child Health J 2015;19:1047-1059.

66 Lindsay KL, Heneghan C, McNulty B, Brennan L, McAuliffe FM: Lifestyle and dietary habits of an obese pregnant cohort. Matern Child Health J 2015;19:25-32.

67 Groh CJ: Poverty, mental health and women: implications for psychiatric nurses in primary care settings. J Am Psychiatr Nurses Assoc 2007;13:267-274.

68 Adler NE, Stewart J: Preface to the biology of disadvantage: socioeconomic status and health. Ann N Y Acad Sci 2010;1186:1-4.

69 Vaz Jdos S, Kac G, Emmett P, Davis JM, Golding J, Hibbeln JR: Dietary patterns, n-3 fatty acids intake from seafood and high levels of anxiety symptoms during pregnancy: findings from the Avon Longitudinal Study of Parents and Children. PLoS One 2013;8: e67671.

70 Golding J, Steer C, Emmett P, Davis JM, Hibbeln JR: High levels of depressive symptoms in pregnancy with low omega- 3 fatty acid intake from fish. Epidemiology 2009;20:598603.

71 Goncalves S, Freitas F, Freitas-Rosa MA, Machado BC: Dysfunctional eating behaviour, psychological well-being and adaptation to pregnancy: a study with women in the third trimester of pregnancy. J Health Psychol 2015;20:535-542.

72 Vilela AA, Farias DR, Eshriqui I, Vaz Jdos S, Franco-Sena AB, Castro MB, et al: Prepregnancy healthy dietary pattern is inversely associated with depressive symptoms among pregnant Brazilian women. J Nutr 2014;144: $1612-1618$
73 Chatzi L, Melaki V, Sarri K, Apostolaki I, Roumeliotaki T, Georgiou V, et al: Dietary patterns during pregnancy and the risk of postpartum depression: the mother-child 'Rhea' cohort in Crete, Greece. Public Health Nutr 2011;14:1663-1670.

74 Laraia B, Vinikoor-Imler LC, Siega-Riz AM: Food insecurity during pregnancy leads to stress, disordered eating, and greater postpartum weight among overweight women. Obesity (Silver Spring) 2015;23:1303-1311.

75 Kendall A, Olson CM, Frongillo EA Jr: Relationship of hunger and food insecurity to food availability and consumption. J Am Diet Assoc 1996;96:1019-1024; quiz 1025-1026.

76 Su KP, Huang SY, Chiu TH, Huang KC, Huang CL, Chang HC, et al: Omega-3 fatty acids for major depressive disorder during pregnancy: results from a randomized, double-blind, placebo-controlled trial. J Clin Psychiatry 2008;69:644-651.

77 Keenan K, Hipwell AE, Bortner J, Hoffmann A, McAloon R: Association between fatty acid supplementation and prenatal stress in African Americans: a randomized controlled trial. Obstet Gynecol 2014;124:1080-1087.

78 Oaks BM, Laugero KD, Stewart CP, Adu-Afarwuah S, Lartey A, Ashorn P, et al: Late-pregnancy salivary cortisol concentrations of Ghanaian women participating in a randomized controlled trial of prenatal lipid-based nutrient supplements. J Nutr 2016;146:343352.

79 Stewart CP, Oaks BM, Laugero KD, Ashorn U, Harjunmaa U, Kumwenda C, et al: Maternal cortisol and stress are associated with birth outcomes, but are not affected by lipidbased nutrient supplements during pregnancy: an analysis of data from a randomized controlled trial in rural Malawi. BMC Pregnancy Childbirth 2015;15:346.

80 Thomas M, Vieten C, Adler N, Ammondson I, Coleman-Phox K, Epel E, et al: Potential for a stress reduction intervention to promote healthy gestational weight gain: focus groups with low-income pregnant women. Womens Health Issues 2014;24:e305-e311.

81 Koletzko B, Lien E, Agostoni C, Bohles H, Campoy C, Cetin I, et al: The roles of longchain polyunsaturated fatty acids in pregnancy, lactation and infancy: review of current knowledge and consensus recommendations. J Perinat Med 2008;36:5-14.

82 Koletzko B, Boey CC, Campoy C, Carlson SE, Chang N, Guillermo-Tuazon MA, et al: Current information and Asian perspectives on long-chain polyunsaturated fatty acids in pregnancy, lactation, and infancy: systematic review and practice recommendations from an early nutrition academy workshop. Ann Nutr Metab 2014;65:49-80. 